WTC2005-63539

\title{
DRAFT
}

\section{ANALYSIS OF BLACK BEARING BALLS FROM A SPACE SHUTTLE BODY FLAP ACTUATOR}

\author{
Kenneth W. Street \\ NASA Glenn Research Center \\ MS 23-2 \\ 21000 Brookpark Road \\ Cleveland $\mathrm{OH} 44135$
}

\author{
Marjorie F. Sovinski \\ NASA Goddard Space Flight Center \\ Code 541 \\ Bldg 30, Rm 112 \\ Greenbelt $\mathrm{OH} 20771$
}

\begin{abstract}
A significantly deteriorated ball bearing mechanism from a body flap actuator on Space Shuttle OV-103 was disassembled and the balls submitted for analysis in conjunction with Return to Flight activities. The OV-103 balls, referred to as the "black balls", were subjected to X-ray photoelectron spectroscopy (XPS), Fourier transform infrared (FT-IR) and Raman micro spectroscopy, surface profilometry, and optical and electron microscopy. The spectroscopic results in combination with microscopy analysis allowed a determination of the lubricant degradation pathway. The chemical attack mechanism does not adequately explain the unique visual appearance of the black balls. Numerous efforts have unsuccessfully focused on duplication of the phenomena causing this unique surface structure and appearance of the black balls. Further detail will be presented supporting these conclusions along with plausible explanations of the unique black appearance to the balls.
\end{abstract}

\section{INTRODUCTION}

The space shuttle body flap contains four actuators to drive it. Four similar actuators in the tail drive the rudder and speed break. These actuators are deemed as Critical 1 components where failure means loss of vehicle and crew. These components are connected in series, hence failure of one actuator in a section of the craft causes complete loss of function in that section of the craft. To date many actuators that have seen service and have been disassembled for maintenance and refurbishment. Only one bearing from all of the actuators examined thus far has the unique appearance of the "black balls". The appearances of the balls from this bearing are best described as black mirrors.

A large number of techniques are available for the elucidation of problems encountered in tribology. Both qualitative techniques such as visual microscopy and quantitative techniques like the spectroscopic ones when combined provide a great amount of information regarding the physical and chemical conditions leading to the end of useful life of mechanical components. We have employed a variety of microscopic, surface topographical and spectroscopic techniques to examine the black balls from OV-103 in order to determine what conditions lead to the unique appearance.

\section{RESULTS AND DISCUSSION}

The as received balls (both black balls and used balls from a body flap actuator on OV-104) had been solvent cleaned with Fluoroclean TM cleaning solvent. No further details were provided on the Black Balls and the used balls. It is unfortunate that none of the extract was maintained for chemical analysis which might have indicated degradation of the lubricant itself. These bearings were previously lubricated with Braycote 601 which containes a perfluoropolyether (PFPE) base oil, Bray $815 \mathrm{Z}$ oil. New balls from stock were sonicated in clean Freon 113 for 3 minutes prior to taking measurements on them.

Black balls were submitted for micro FT-IR and micro Raman analysis. Spectra were run on all balls at a minimum of three arbitrary locations. All IR spectra clearly showed no lubricant which is consistent with the solvent cleaning applied to the balls prior to submission for analysis. The IR did show weak indications of a thin layer of "End Stage Lubricant" in all 
spectra. The balls were then subjected to Raman analysis which is more definitive for the detection of amorphous graphitic signatures characteristic of end stage degradation. All Raman spectra of the black and used balls showed the characteristic D and $G$ peaks associated with disordered graphitic material. None of the spectra showed any unusual bands. In itself, the appearance of amorphous carbon is not unusual and is the result of normal usage of the lubricant during bearing operation.

Data from the XPS analysis indicated iron fluoride and iron oxides on the black ball (Fig. 1). The color of iron fluoride is dependent on the oxidation state and state of hydration and ranges from white to yellow to green. Metal fluorides and in particular iron fluoride has been shown to catalyze the decomposition of PFPE lubricants. The amount of iron fluoride is key to telling if metal fluoride catalyzed PFPE decomposition was the primary cause of grease degradation. Under the specimen storage and shipping conditions it is likely that the iron fluoride hydrolyzed into iron oxides and hydrofluoric acid (HF). Some iron oxides and mixed hydroxy-oxides are typically black.

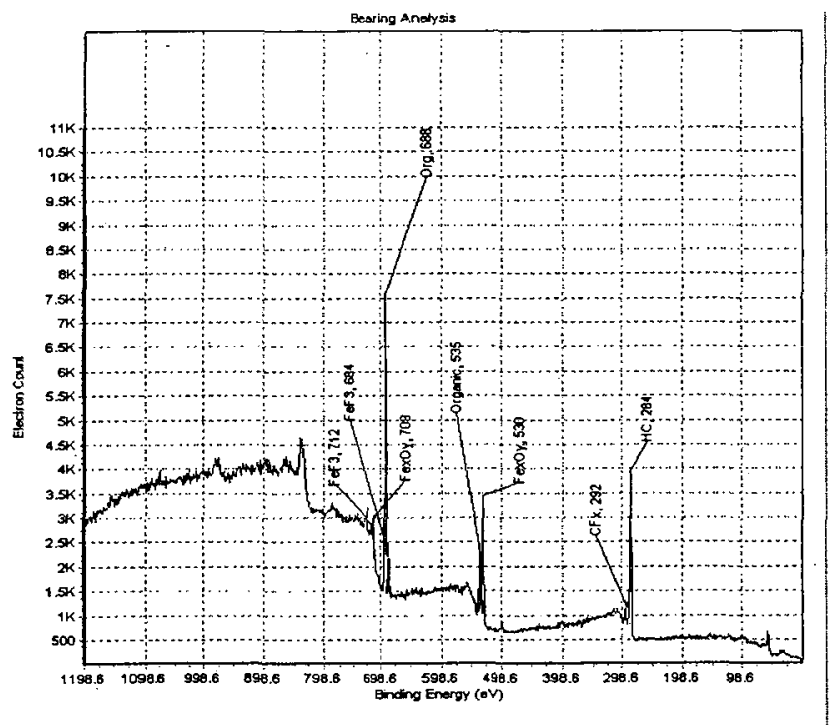

Figure 1. Initial XPS spectrum of black ball.

Photomicrographs indicated significant pitting of the balls as compared to what would be typically seen on a new ball. This was apparent in pictures taken from both optical and electron microscopes (Fig 2). The distinct appearance of grain boundary deterioration (the grainy appearance of the surface in the photomicrographs) may have led to the pitting. The blackened appearance of the black balls is probably from a combination of effects. First, some iron oxides are black. Iron fluoride can hydrolyze to produce iron oxides or hydroxyoxides and HF which would be lost. Second, the pitting of the balls allows light to become effectively trapped in the pits and lost thus resulting in a blackened appearance.
Surface profilometry was performed on the Zygo optical surface profilometer and the data corrected for spherical curvature. It is apparent from the comparison of the three specimens that the black balls have seen significantly more deterioration than the used balls. Chemical pitting is seen evenly distributed over the surface of these balls. The used balls themselves are far from the high grade finish of a pristine ball; however, they still exhibit the appearance of a silvered mirror.

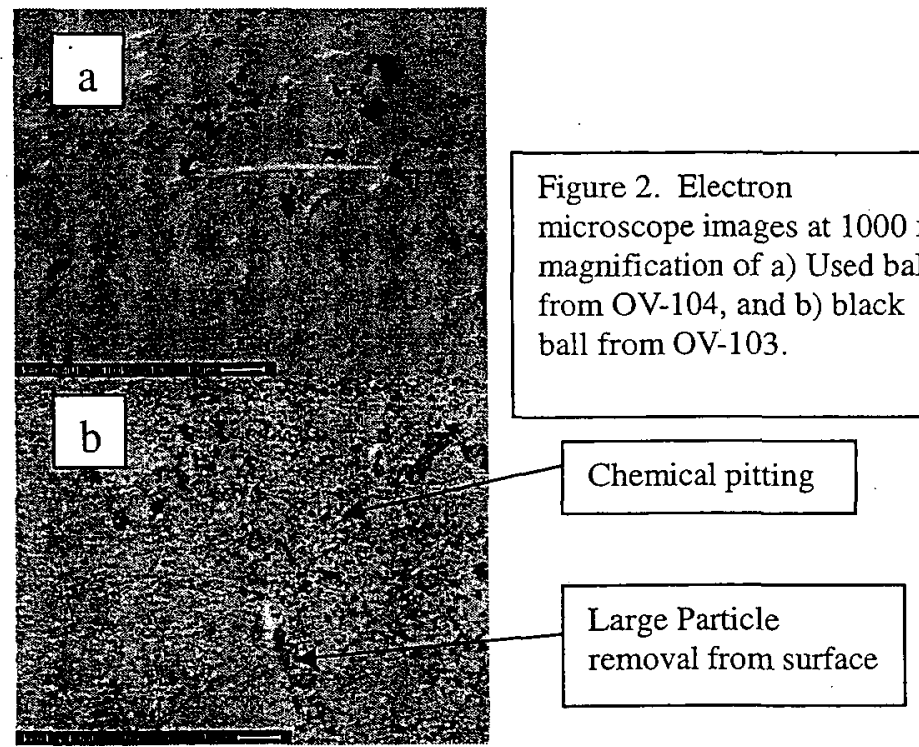

It appears that the Braycote 601 grease decomposed into acid fluorides and HF, both of which corrode most steels. The appearance of iron fluoride is an indication that the process could have been catalytic leading to an accelerated decomposition from the time of generation of the metal fluoride. This process does not occur on steel surfaces rubbed with PFPE except at elevated temperatures[1]. The corrosion appears to have been along the grain boundaries leading to a weakening of the surface structure. Further exercising of the bearing caused surface removal of larger particles on the order of 5 to 10 microns.

\section{ACKNOWLEDGMENTS}

The authors gratefully acknowledge support form Duane Dixon, and John Lucero for their assistance with electron microscopy and surface topography respectively. John Canham is acknowledged for his assistance with the interpretation of the XPS spectra and to William R. Jones for his helpful discussions.

\section{REFERENCES}

[1] Herrera-Fierro, P., Jones, W.R., and Pepper, S.V., 1993, "Interfacial Chemistry of a Perfluoropolyether Lubricant Studied by X-ray Photoelectron Spectroscopy and Temperature Desorption Spectroscopy," J. Vac. Sci. Technol, 11(2), pp.354367. 